\title{
Variation of Urate Transport in the Nephrons in Subtypes of Hyperuricemia
}

\author{
Toru Nakamura a, b Rie Nishi a Tuneo Tanaka a Kazutaka Takagi a \\ Katsuya Sakai b Mihoko Takai a, b Shigeru Morishima b \\ Takahiro Yamauchi a Takanori Ueda a \\ a First Department of Internal Medicine, Faculty of Medical Sciences, Fukui University, \\ Matsuoka, and b Hayashi General Hospital, Echizen, Japan
}

\author{
Key Words \\ Urate transport in nephrons - Urate secretion - Postsecretory urate reabsorption - Urinary \\ urate excretion - Urate underexcretion - Subtypes of hyperuricemia - Benzbromarone . \\ Benzbromarone-loading urate clearance test
}

\begin{abstract}
Background: Hyperuricemia cases (HU) can be classified into four subgroups by combining the two main causes of hyperuricemia, i.e. urate underexcretion and overproduction. These subgroups are as follows: underexcretion-type cases (UE); overproduction-type cases (OP); combined-type cases, and normal-type cases. Since urinary urate excretion (Uua) and urate clearance differ significantly between UE and $O P$, urate transport in the nephrons and the intratubular urate contents might also differ. Such differences might help clarify the pathophysiology of urate underexcretion in subgroups of hyperuricemia, and thus reveal its underlying mechanisms. Methods: Urate transport coefficients in each subtype of HU were determined employing the previously reported benzbromarone-loading urate clearance tests. The subtype cases of HU were plotted on a graph of urate transport coefficients versus Uua as coordinates. The characteristic features in the distribution of subtype cases on graphs were analyzed in relation to Uua. Results: The mean ( \pm standard error) tubular secretion rate (TSR) in the UE $(48.7 \pm 1.7 \mathrm{ml} / \mathrm{min})$ was significantly lower and the postsecretory urate reabsorption rate $\left(R_{2}\right)$ in the UE $(0.904 \pm 0.004)$ was significantly higher than those in the normal controls $(78.0 \pm 2.1 \mathrm{ml} / \mathrm{min}$ and $0.877 \pm 0.003)$ or the OP $(61.1 \pm 3.2 \mathrm{ml} / \mathrm{min}$ and $0.861 \pm 0.009)$. Decrements of TSR and increments of $R_{2}$ in the UE were largest in the subtypes of the HU, in terms of case numbers and the deviation rate of the group. Conversely, decrements of TSR and increments of $R_{2}$ were smallest in the OP. A significant correlation was identified between TSR and Uua ( $r=0.345, p<0.0001)$, and a significant negative correlation was also found between $R_{2}$ and Uua ( $\left.r=-0.393, p<0.0001\right)$. Conclusion: In the UE, hyperuricemia is induced mainly
\end{abstract}


Nakamura et al.: Variation of Urate Transport in the Nephrons in Subtypes of Hyperuricemia

by urate underexcretion, which results from the combination of two main causes in urate transporters of the nephron: significantly lower TSR and significantly higher $\mathrm{R}_{2}$. Neither of these was observed in OP. Differences in urate transporters in subtypes of the $\mathrm{HU}$ might be important not only for understanding the pathophysiology and mechanisms of urate underexcretion and hyperuricemia, but also for providing a strategic therapy for hyperuricemia.

(C) 2013 S. Karger AG, Basel

\section{Introduction}

Hyperuricemia is induced by two main abnormalities in urate metabolism, namely urate underexcretion and urate overproduction [1-5]. In hyperuricemia cases (HU), underexcretion-type cases (UE), which are characterized by significantly lower urate clearance (Cua) and overproduction-type cases (OP), which are characterized by higher urinary urate excretion (Uua), can be classified by the Cua test $[5,6]$. Since urate is mainly excreted from the nephrons, considerable differences might be found in urate transport in the nephrons between UE and OP, reflecting the differences in urate metabolism between these types. To understand the pathophysiology of urate underexcretion in HU in greater detail, we have investigated quantitative estimation of urate transport in the nephrons employing the benzbromarone (BBR)-loading urate clearance test [7, 8]. Significant differences have previously been noted in urate transport between HU and normal controls (NC) [7], but more marked differences in urate transport were found between UE and OP. Based on these findings, the relationship between urate transport and Uua and the mechanisms underlying urate underexcretion in HU, particularly in UE, were investigated. Differences in the pathophysiology of urate underexcretion between UE and OP imply the possibility for rational treatment of hyperuricemia based on the specific subtype.

\section{Materials and Methods}

This present study was approved by the ethics committee at Hayashi General Hospital (Director, Dr. M. Nojiri). The participants comprised 172 male gouty patients (age range, 22-82 years) with hyperuricemia, with a serum urate concentration (Sua) $>7.0 \mathrm{mg} / \mathrm{dl}$, along with 30 male volunteers (age range, 19-69 years) who had provided informed consent to act as NC. Prior to examination, administration of medicines affecting Sua or Uua, such as BBR, allopurinol, febuxostate [9, 10], bucolome, losartan, diuretics, fenofibrate, or nucleoside derivatives, was discontinued unless otherwise indicated. All patients provided written informed consent prior to enrollment. Cua, creatinine clearance (Ccr) and Cua determined after administering $100 \mathrm{mg}$ of BBR [CuaBBR(100)] were determined using the BBR-loading urate clearance test as reported previously $[7,8]$. Urate transport coefficients such as urate glomerular filtration (UGF), urate reabsorption, urate reabsorption rate $\left(\mathrm{R}_{1}\right)$, urate tubular secretion (UTS), tubular secretion rate (TSR), urate postsecretory reabsorption $\left(\mathrm{UR}_{2}\right)$ and urate postsecretory reabsorption rate $\left(\mathrm{R}_{2}\right)$ as well as calculated urinary urate excretion (Uex) were calculated as reported previously [7].

\section{Classification of Hyperuricemia Subtypes}

Since the main causes of hyperuricemia are considered to be underexcretion and overproduction in urate metabolism of human subjects, we classified HU into 4 subtypes according to the combination of underexcretion and overproduction, as determined by the urate clearance test $[5,6]$. UE were defined as cases in which Cua was significantly lower than the 
Nakamura et al.: Variation of Urate Transport in the Nephrons in Subtypes of Hyperuricemia

Table 1. Urate transport in the nephrons and its urinary excretion in each subtype of hyperuricemia

\begin{tabular}{|c|c|c|c|c|c|c|c|c|c|c|c|c|c|}
\hline \multirow[t]{2}{*}{ Type } & \multirow{2}{*}{$\begin{array}{l}\text { Cases, } \\
\text { n (\%) }\end{array}$} & \multicolumn{6}{|c|}{ BBR-loading urate clearance tests } & \multicolumn{5}{|c|}{ Approximate urate transports in nephrons } & \multirow[t]{2}{*}{ Uex } \\
\hline & & Sua & Uua & Cua & $\mathrm{Ccr}$ & $\mathrm{R}$ & $\begin{array}{l}\text { CuaBBR } \\
(100)\end{array}$ & UGF & UTS & TSR & $\mathrm{UR}_{2}$ & $\mathrm{R}_{2}$ & \\
\hline \multirow[t]{3}{*}{ NC } & 30 & 53.1 & 7.84 & 9.76 & 133 & 7.34 & 48.4 & 7,060 & 4,130 & 78.0 & 3,680 & 0.877 & 520 \\
\hline & & 6.8 & 1.06 & 1.28 & 16 & 1.23 & 7.1 & 1,240 & 800 & 11.6 & 750 & 0.017 & 70 \\
\hline & & 1.2 & 0.19 & 0.23 & 2.9 & 0.22 & 1.3 & 230 & 150 & 2.1 & 140 & 0.003 & 13 \\
\hline \multirow[t]{3}{*}{$\mathrm{HU}$} & 172 & 86.6 & 7.20 & 5.73 & 109 & 5.24 & 33.8 & 9,440 & 4,480 & 51.8 & 4,080 & 0.892 & 490 \\
\hline & (100) & 12.0 & 2.27 & 1.88 & 32 & 1.57 & 12.1 & 2,700 & 1,500 & 18.8 & 1,440 & 0.044 & 150 \\
\hline & & 0.9 & 0.17 & 0.14 & 2.4 & 0.12 & 0.9 & 210 & 110 & 1.4 & 110 & 0.003 & 11 \\
\hline \multirow[t]{3}{*}{ UE } & 117 & 86.6 & 5.98 & 4.77 & 100 & 4.78 & 31.8 & 8,650 & 4,220 & 48.7 & 3,890 & 0.904 & 415 \\
\hline & $(68)$ & 11.3 & 1.27 & 1.18 & 30 & 1.41 & 11.9 & 2,570 & 1,460 & 18.6 & 1,430 & 0.043 & 90 \\
\hline & & 1.1 & 0.12 & 0.11 & 2.8 & 0.14 & 1.1 & 240 & 140 & 1.7 & 130 & 0.004 & 10 \\
\hline \multirow[t]{3}{*}{$\mathrm{OP}$} & 28 & 83.3 & 10.84 & 8.71 & 136 & 6.42 & 40.0 & 11,330 & 5,100 & 61.1 & 4,490 & 0.861 & 725 \\
\hline & $(16)$ & 8.6 & 1.50 & 0.97 & 26 & 1.44 & 10.8 & 2,350 & 1,260 & 16.8 & 1,090 & 0.050 & 90 \\
\hline & & 1.6 & 0.28 & 0.18 & 4.9 & 0.27 & 2.0 & 440 & 240 & 3.2 & 210 & 0.009 & 40 \\
\hline \multirow[t]{3}{*}{$\mathrm{C}$} & 17 & 97.1 & 8.84 & 6.13 & 112 & 5.47 & 34.1 & 10,870 & 5,070 & 52.2 & 4,580 & 0.885 & 600 \\
\hline & $(10)$ & 16.7 & 0.95 & 1.06 & 21 & 1.32 & 11.3 & 2,320 & 1,660 & 17.8 & 1,660 & 0.039 & 60 \\
\hline & & 4.1 & 0.23 & 0.26 & 5.0 & 0.32 & 2.7 & 560 & 400 & 4.3 & 400 & 0.010 & 15 \\
\hline \multirow[t]{3}{*}{$\mathrm{N}$} & 10 & 77.3 & 8.20 & 7.74 & 136 & 5.71 & 38.3 & 10,510 & 4,510 & 58.4 & 4,020 & 0.871 & 595 \\
\hline & $(6)$ & 4.6 & 0.39 & 0.40 & 20 & 0.79 & 13.8 & 1,460 & 1,700 & 22.3 & 1,670 & 0.051 & 40 \\
\hline & & 1.5 & 0.12 & 0.13 & 6.3 & 0.25 & 4.4 & 460 & 540 & 7.0 & 540 & 0.016 & 10 \\
\hline
\end{tabular}

Upper value = Mean; middle value $=$ standard deviation; lower value $=$ standard error. The following units of measurement apply: Sua, $\mu \mathrm{g} / \mathrm{ml}$; Uua, $\mu \mathrm{g} / \mathrm{kg} / \mathrm{min}$; Cua, $\mathrm{ml} / \mathrm{min} / 1.73 \mathrm{~m}^{2}$; Ccr, $\mathrm{ml} / \mathrm{min} / 1.73 \mathrm{~m}^{2}$; CuaBBR(100), $\mathrm{ml} / \mathrm{min} / 1.73$ m² UGF, $\mu \mathrm{g} / \mathrm{min}$; $\mathrm{UTS}, \mu \mathrm{g} / \mathrm{min}$; TSR, $\mathrm{ml} / \mathrm{min} ; \mathrm{UR}_{2}, \mu \mathrm{g} / \mathrm{min} ; \mathrm{Uex}, \mu \mathrm{g} / \mathrm{min}$.

normal range (i.e. $<7.3 \mathrm{ml} / \mathrm{min}$ ) $[5,6]$. OP corresponded to those patients in whom Uua was higher than $(0.43 \mathrm{Cua}+5.43) \mu \mathrm{g} / \mathrm{min}$, because OP determined by the labeled urate tracer technique were roughly separated from UE by the line Uua $=(0.43 \mathrm{Cua}+5.43) \mu \mathrm{g} / \mathrm{min}$ in the Cua-versus-Uua plot $[11,12]$, and in whom Cua was higher than $7.3 \mathrm{ml} / \mathrm{min}$. The 4 subtypes of HU were UE, OP, combined-type cases (C) and normal-type cases (N). HU classified as N had both Cua within the normal range and Uua below the criterion line from urate clearance tests. N were characterized by a low-grade elevation of Sua and a short duration of morbidity, representing patients in the early stage of hyperuricemia. Such cases might change to other types of HU during the clinical course.

\section{Results}

Urate Transport Coefficients by HU Subtype

Significant differences in the urate transport coefficients were noted between the HU and the NC [7], but more marked differences were found between the UE and the OP (table 1). In terms of urate transport, the UE were characterized by the lowest TSR and UTS, by the highest $\mathrm{R}_{2}$, and subsequently by the lowest Uex in the subtypes of HU. Conversely, OP were characterized by the highest TSR and UTS, by the lowest $\mathrm{R}_{2}$, and subsequently by the highest Uex. Accordingly, the marked difference in Uua between UE and OP was attributed to TSR and $R_{2}$ in the subgroups. 
Nakamura et al.: Variation of Urate Transport in the Nephrons in Subtypes of Hyperuricemia

Table 2. Correlation coefficients of urate transporters and clinical parameters

\begin{tabular}{|c|c|c|c|c|c|c|c|c|c|c|c|}
\hline & Sua & Uua & Cua & $\mathrm{Ccr}$ & $\mathrm{R}$ & UGF & UTS & TSR & $\mathrm{UR}_{2}$ & $\mathrm{R}_{2}$ & Uex \\
\hline Sua & & -0.084 & -0.633 & -0.421 & -0.290 & +0.314 & +0.101 & -0.510 & +0.116 & +0.103 & +0.014 \\
\hline Uua & & & +0.757 & +0.506 & +0.426 & +0.473 & +0.344 & +0.345 & +0.269 & -0.393 & +0.939 \\
\hline Cua & & & & +0.666 & +0.507 & +0.196 & +0.205 & +0.593 & +0.135 & -0.368 & +0.720 \\
\hline $\mathrm{Ccr}$ & & & & & -0.232 & +0.712 & +0.280 & +0.486 & +0.246 & -0.151 & +0.536 \\
\hline $\mathrm{R}$ & & & & & & -0.480 & -0.070 & +0.162 & -0.120 & -0.356 & +0.355 \\
\hline UGF & & & & & & & +0.376 & +0.102 & +0.352 & -0.078 & +0.584 \\
\hline UTS & & & & & & & & +0.773 & +0.933 & +0.607 & +0.388 \\
\hline TSR & & & & & & & & & +0.756 & +0.430 & +0.315 \\
\hline $\mathrm{UR}_{2}$ & & & & & & & & & & +0.655 & +0.310 \\
\hline $\mathrm{R}_{2}$ & & & & & & & & & & & -0.380 \\
\hline
\end{tabular}

Regarding urate secretion in the nephrons, UTS in the HU, including in its subtypes, was generally higher than that in NC. The increment of UTS in the UE was minimal in the subtypes of the HU, and UTS in the UE was nearly equal to that in the NC. Although UTS correlated highly with TSR ( $r=0.733, p<0.0001)$, as shown in table 2, TSR in all subtypes of the HU was significantly lower than that in the NC and that in the UE was lowest in the subtypes of the HU. Significant correlation was observed between TSR and Uua ( $r=0.345, p<0.0001$ ) (table 2). Furthermore, the significant correlation between TSR and Cua ( $r=0.593)$ was markedly higher than that between UTS and Cua $(r=0.205)$. Since UE were defined as HU with significantly lower Cua than that in $\mathrm{NC}$, urate excretion efficiency in the UE was significantly low, and TSR was considered to be highly correlated with urate excretion and to offer a better parameter than UTS for evaluating Uua. UTS in the OP was markedly higher $(+23.5 \%)$ than that in the NC and was highest in the subtypes of the HU. These findings suggest that UTS is highly related to urate excretion and possibly related to urate production, because urate metabolism is considered to be in an increased steady state.

Regarding postsecretory reabsorption of urate, $\mathrm{R}_{2}$ was significantly higher in the UE than in the NC. Increment of $\mathrm{R}_{2}$ compared to the $\mathrm{NC}$ was highest in the UE $(+3.2 \%)$ and lowest in the OP $(-1.7 \%)$ in the subtypes of the HU. A significant negative correlation was obtained between $R_{2}$ and Uua $(r=-0.393, p<0.0001)$ and also between $R_{2}$ and Cua $(r=-0.368, p<$ 0.0001 ) (table 2 ), suggesting that increases in $R_{2}$ correlate with decreased urate excretion. $R_{2}$ was highly correlated with $\mathrm{UR}_{2}(\mathrm{r}=0.655, \mathrm{p}<0.0001)$, as shown in table 2 , and $\mathrm{UR}_{2}$ in all subtypes of the $\mathrm{HU}$ was also generally higher than that in NC. Compared to the NC, $\mathrm{UR}_{2}$ was $+6.0 \%$ higher in the UE and $+22.0 \%$ higher in the OP. Although $\mathrm{UR}_{2}$ was higher in the OP than in the UE, Uua was significantly higher in the OP than in the UE. This is probably because UTS in the OP was very much higher than postsecretory reabsorption. The ratio of $\mathrm{UR}_{2} / \mathrm{UTS}$ was lower in the OP than in the UE. TSR and $\mathrm{R}_{2}$ in the $\mathrm{HU}$ with the combination of underexcretion and overproduction (C) were midway between those for the UE and the OP. Subsequently, Uex in the $C$ was also midway between values in the UE and the OP.

Estimation of Intratubular Urate Contents in the Nephrons of the Subtypes of HU (fig. 1)

UGF in all subtypes of the HU was generally higher than that in the NC, and UGF in the OP was markedly higher than that in the UE, but almost all the filtrated urate was thought to be reabsorbed at the urate reabsorption stage. Therefore, intratubular urate contents in all subtypes might be minimal and might not differ significantly. TSR in all subtypes was generally lower than that in the NC, but UTS in all subtypes returned back to NC levels or higher. Intratubular urate contents in all subtypes were thus considered to be higher than those of the NC until the stage 
Nakamura et al:: Variation of Urate Transport in the Nephrons in Subtypes of Hyperuricemia

\begin{tabular}{|c|c|c|c|c|c|c|c|}
\hline \multicolumn{2}{|c|}{$\begin{array}{l}\text { urate } \\
\text { transport }\end{array}$} & $\begin{array}{l}\mathrm{NC} \\
\text { intratubular } \\
\text { urate } \\
\text { contens } \\
\text { ( } \mathrm{g} / \mathrm{min})\end{array}$ & & $\begin{array}{l}\mathrm{HU} \\
\text { intratubula } \\
\text { urate } \\
\text { contens } \\
\text { ( } \mathrm{g} / \mathrm{min})\end{array}$ & & $\begin{array}{l}\text { UE } \\
\text { intratubular } \\
\text { urate } \\
\text { contens } \\
\text { ( } \mu \mathrm{g} / \mathrm{min})\end{array}$ & $\begin{array}{l}\text { OP } \\
\text { intratubular } \\
\text { urate } \\
\text { contens } \\
\text { ( } \mathrm{g} / \mathrm{min})\end{array}$ \\
\hline UGF & 7,060 (100\%) & 7,060 & 9,440 (100\%) & 9,440 & $8,650(100 \%)$ & 8,75 & $11,330(100 \%)$ \\
\hline $\mathrm{UR}_{1}$ & \multicolumn{2}{|c|}{$6,990(99 \%) \longleftarrow$} & $9,350(99 \%)$ & $\longleftarrow$ & $8,565(99 \%)$ & & $11,215(99 \%) \longleftarrow_{115}$ \\
\hline UTS & \multicolumn{3}{|c|}{$4,130(58 \%) \underset{4,200}{\longrightarrow}$} & $\overrightarrow{4,570}$ & $4,220(49 \%)$ & $\overrightarrow{4,305}$ & $5,100(45 \%) \longrightarrow \underset{5,215}{\longrightarrow}$ \\
\hline \multirow[t]{2}{*}{$\mathrm{UR}_{2}$} & $\begin{array}{l}3,680(52 \%) \\
R_{2}=0.877\end{array}$ & $\longleftarrow$ & $\begin{array}{l}4,080(43 \%) \\
R_{2}=0.892\end{array}$ & & $\begin{array}{c}3,890(45 \%) \\
R_{2}=0.904\end{array}$ & 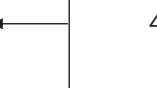 & $\begin{array}{c}4,490(40 \%) \\
R_{2}=0.861\end{array}$ \\
\hline & Uex & $520^{\star}(7.4 \%)$ & & $490(5.2 \%)$ & & 415 (4.9\%) & 725 (6.4\%) \\
\hline
\end{tabular}

Fig. 1. Comparison of intratubular urate contents between $\mathrm{UE}$ and $\mathrm{OP}$. $\mathrm{UR}_{1}=$ Urate reabsorption.

of UTS, but were reduced by reabsorption with significantly higher $\mathrm{R}_{2}$ in HU subtypes at the stage of $U_{2}$, especially in the UE with markedly higher $R_{2}$, resulting in significantly lower Uex. In the OP, intratubular urate contents were markedly higher than those in the $\mathrm{NC}$ at the stage of UTS. Furthermore, $\mathrm{R}_{2}$ in the OP was significantly lower than that in the $\mathrm{NC}$, and $\mathrm{UR}_{2}$ in the OP was relatively lower. Subsequently, Uex in the OP was markedly higher than that in the NC. Accordingly, a marked difference in Uua was evident between the UE and the OP. These findings indicate that both TSR and $\mathrm{R}_{2}$ are important factors for determining urate excretion efficacy.

\section{Correlation between Urate Transport Coefficients (table 2)}

Sua has been used as a primary parameter for differential diagnosis and understanding the urate metabolism underlying hyperuricemia in clinical practice for gouty patients; however, correlation coefficients between Uua and urate transport coefficients were considered to be more valuable than those between Sua and urate transport coefficients for investigating the pathophysiology of intratubular urate contents and mechanisms of urate underexcretion in hyperuricemia, because the former were generally higher than the latter. Among the correlation coefficients between Cua and urate transport coefficients, TSR and $\mathrm{R}_{2}$ (negative) were found to show higher correlations than the others, both of which might be valuable transporters for determining Uua, but might not be related to UGF, because correlation coefficients of UGF and TSR as well as those of UGF and $\mathrm{R}_{2}$ were low. Ccr showed a significantly higher correlation with TSR, but a lower one with $\mathrm{R}_{2}$. High-grade correlation coefficients were obtained between UTS and TSR ( $r=0.773, p<0.0001)$, both of which are closely related to urate secretion, and UTS was calculated as a product of TSR and Sua [7]. A relationship similar to that between UTS and TSR was also seen for $\mathrm{UR}_{2}$ and $\mathrm{R}_{2}(r=0.655$, $\mathrm{p}<0.0001$ ), both of which are closely related to urate postsecretory reabsorption, and $U_{2}$ is a product of (0.01 UGF + UTS) and $R_{2}$ [7]. High-grade correlation coefficients were also obtained between UTS and $\mathrm{UR}_{2}(\mathrm{r}=0.993, \mathrm{p}<0.0001)$, which was higher than the correlation coefficient between $R_{2}$ and $U_{2}$, suggesting that $U_{2}$ was much more influenced by UST than by $R_{2}$. This is why correlation coefficients between $U R_{2}$ and $U u a(r=0.269, p<0.0001)$ were not negative, in contrast to the common concept that larger $U_{2}$ would result in smaller Uua. 


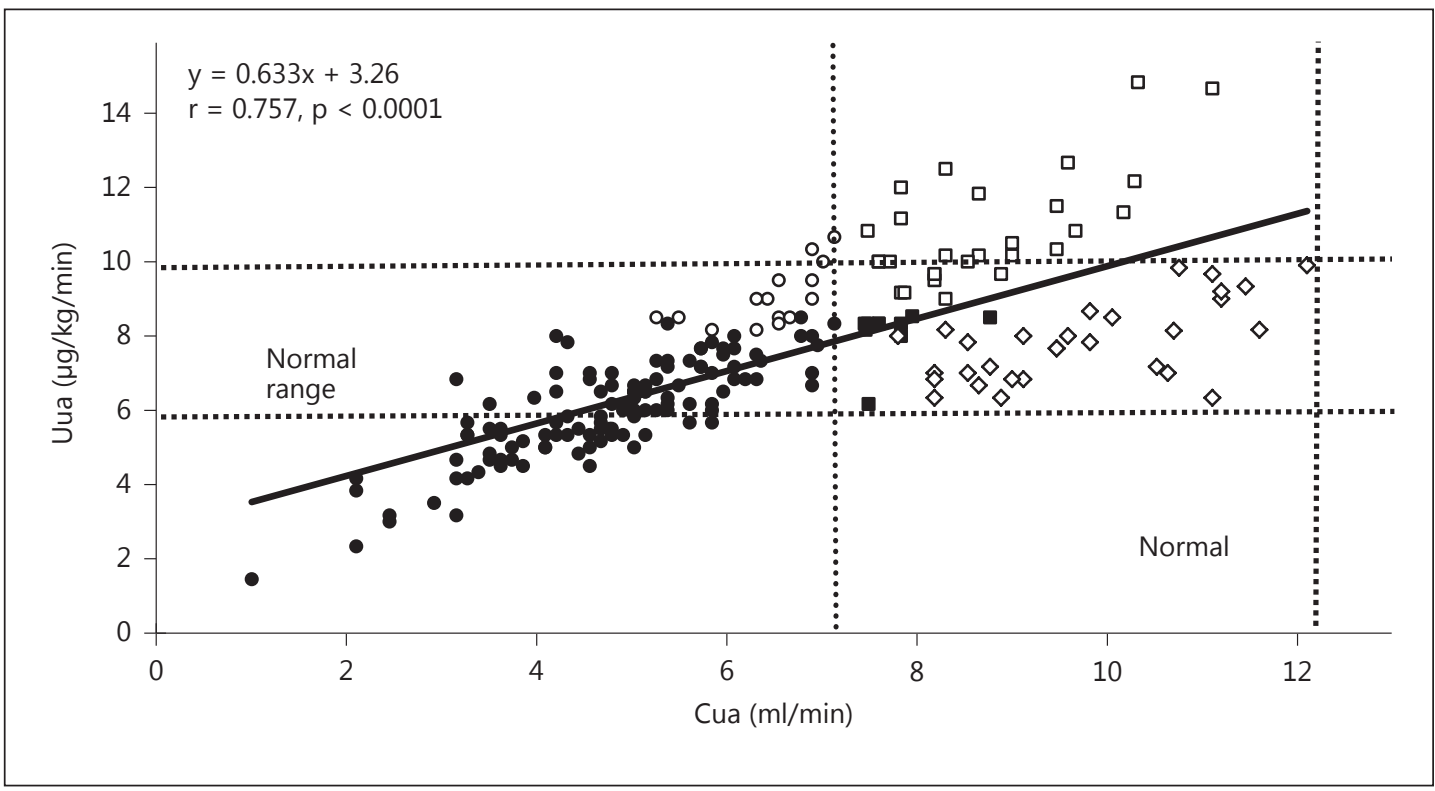

Fig. 2. Distribution of each type of HU on a plot evaluating Uua as a function of Cua. $\bigcirc=\mathrm{UE} ; \square=\mathrm{OP} ; \mathrm{O}=\mathrm{C}$; $\mathbf{\square}=\mathrm{N} ; \diamond=\mathrm{NC} ;----=$ regression equation and line.

Distribution of HU Subtypes on Graphs of Uua versus Cua as Coordinates (fig. 2)

The OP were mainly located in the area of significantly higher Uua and the normal Cua. Conversely, UE were located in the significantly lower Cua range, but not all UE were located in the significantly lower Uua range area. Only approximately $1 / 3$ of the UE were located in the significantly lower Uua range, with $2 / 3$ in the normal Uua range, although UE were defined as cases in which Cua was significantly lower than that in $\mathrm{NC}$, suggesting that the expected decrease in Uua with decreased Cua was restored by increased Sua in the UE. The C and the $\mathrm{N}$ were located in border areas between the distribution of UE and OP. Since the UE and the OP showed great differences in the distribution on the graph of Uua versus Cua as coordinates, together with differences in urate transport coefficients, the distribution of the UE and the OP on graphs of urate transport coefficients versus Uua as coordinates was investigated to provide a better understanding of the pathophysiology and mechanisms of urate underexcretion in hyperuricemia.

\section{Distribution of HU Subtypes on Graphs of Urate Transport Coefficients versus Uua as Coordinates \\ UGF-versus-Uua Graph (fig. 3)}

As a whole, a significant correlation was obtained between UGF and Uua ( $\mathrm{r}=0.473, \mathrm{p}<$ 0.0001 ). The distribution of UE was spread widely in the horizontal (UGF) direction and mainly located in the normal to the higher UGF range, but was limited in a relatively narrow area in the vertical (Uua) direction, that is the lower half of the normal Uua range and the upper part of the lower Uua range. Approximately $40 \%$ of the UE were located in the significantly higher UGF range, but the majority of the UE in the high UGF was located within the normal range of Uua. The Uua/UGF ratio in UE was lower than that in NC, suggesting that urate underexcretion in UE might be functioning in the lower areas of UGF in the intratubular urate flow. The OP were located mainly in the higher UGF and the higher Uua area. The Uua/UGF ratio was not decreased in the OP. There were only 7 cases (6.0\%) of all 117 UE in the significantly lower UGF and lower Uua range, in which low UGF could be explained as a direct cause of urate underexcretion. 


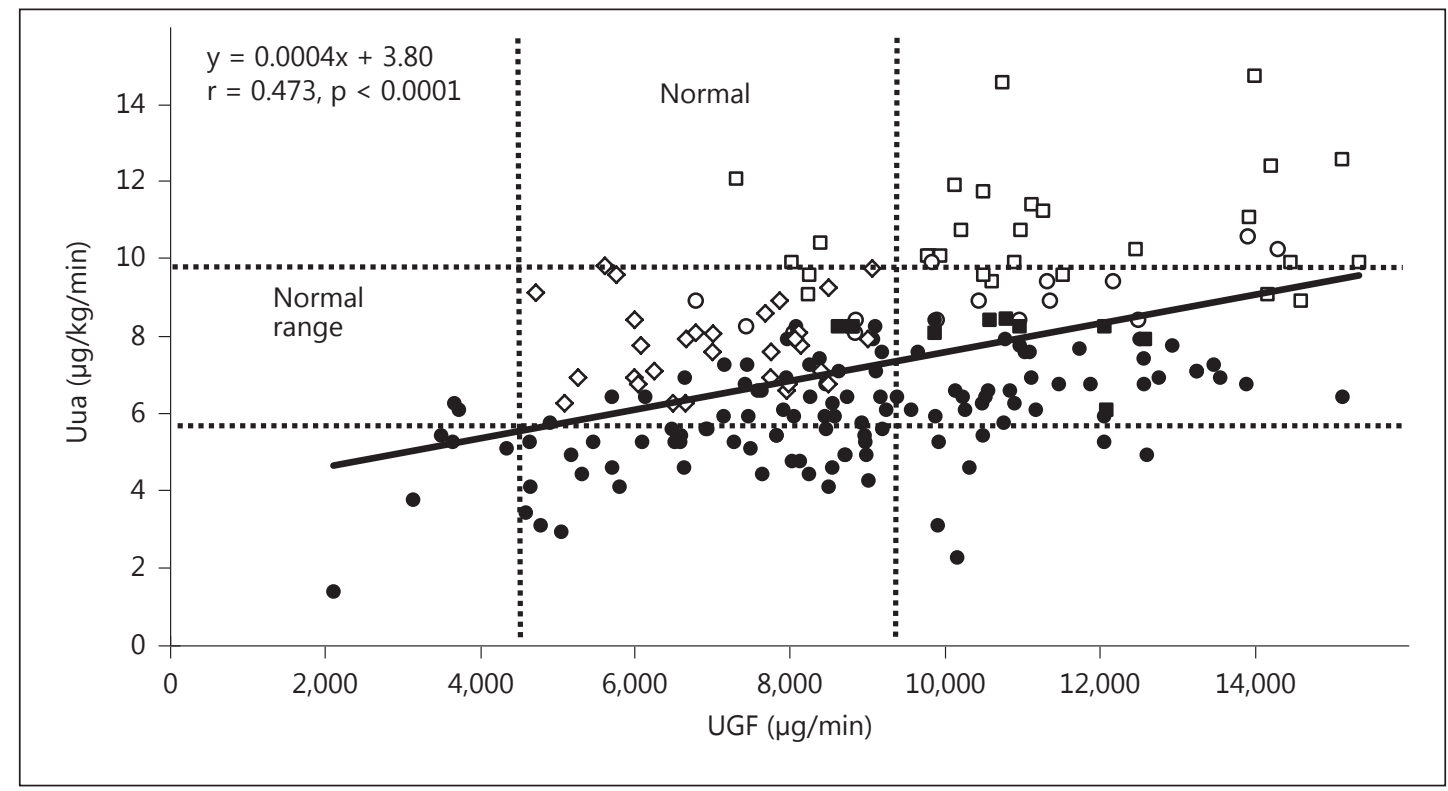

Fig. 3. Distribution of each type of HU on a plot evaluating Uua as a function of UGF. $\bigcirc=\mathrm{UE} ; \square=\mathrm{OP} ; \mathrm{O}=\mathrm{C}$; $\mathbf{\square}=\mathrm{N} ; \diamond=\mathrm{NC} ;----=$ regression equation and line.

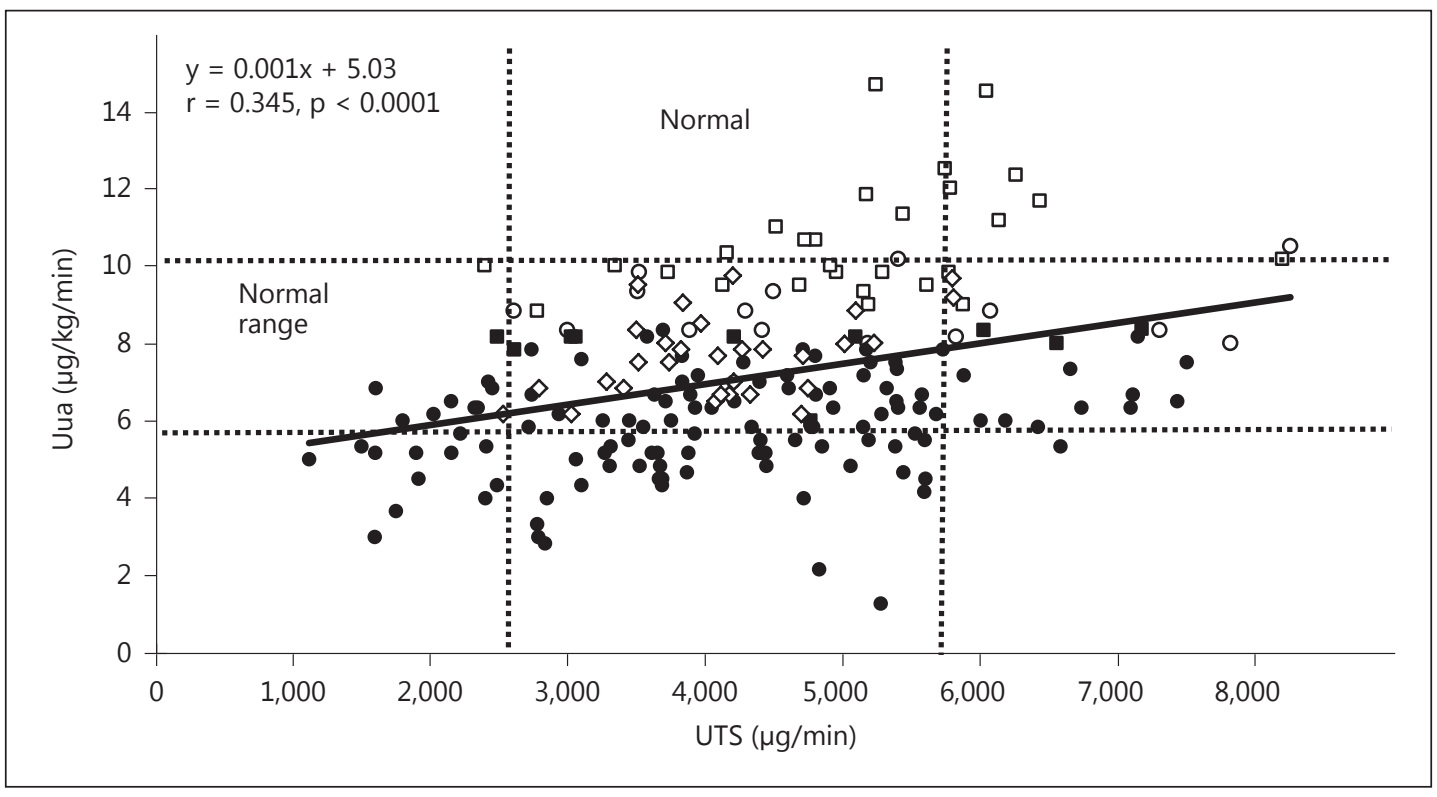

Fig. 4. Distribution of each type of HU on a plot evaluating Uua as a function of UTS. $\bigcirc=U E ; \square=O P ; O=C$; $\mathbf{\square}=\mathrm{N} ; \diamond=\mathrm{NC} ;----=$ regression equation and line.

UTS-versus-Uua Graph (fig. 4)

A significant correlation was obtained between UTS and Uua $(r=0.345, p<0.0001)$. The distribution of the UE was spread in the horizontal (UTS) direction and was in the lower to the higher UTS range, but was limited to a narrow area in the vertical (Uua) direction. The majority $(73 \%)$ of UE was located in the normal UTS range, and the distribution could be 


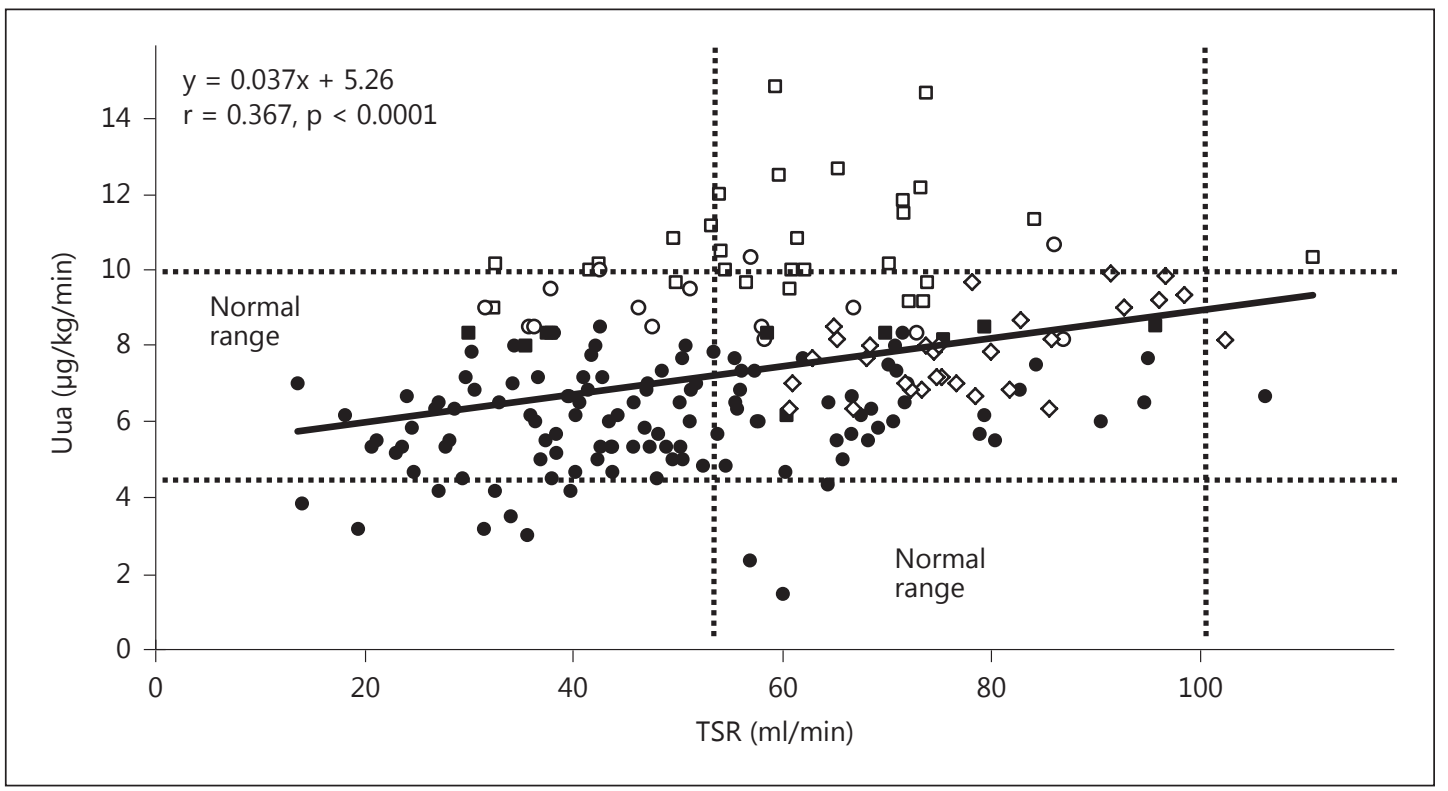

Fig. 5. Distribution of each type of HU on a plot evaluating Uua as a function of TSR. $\bigcirc=U E ; \square=O P ; O=C$; $\mathbf{\square}=\mathrm{N} ; \diamond=\mathrm{NC} ;----=$ regression equation and line.

roughly shifted vertically to a lower Uua compared to the NC. That is, approximately half of these UE in the normal UTS range were shown to have significantly lower Uua. Accordingly, the Uua/UTS ratio in these UE was lower than that in the NC, indicating that Uua in the UE was lower than that in the NC even if UTS in the UE was the same as that in the NC, suggesting that factors other than low UTS might be more significant causes of urate underexcretion. The OP were mainly located in the higher part in the normal UTS range area to the lower part in the higher UTS and the higher Uua range, in which Uua/UTS was higher than in the NC even if UTS was the same between the UE and the NC. There were only 11 UE (9.4\%) located in the significantly lower UTS and lower Uua range, in which low UTS could be explained as a direct cause of urate underexcretion.

TSR-versus-Uua Graph (fig. 5)

A significant correlation was obtained between TSR and Uua in the HU and the NC $(\mathrm{r}=$ $0.367, \mathrm{p}<0.0001$ ). Approximately $2 / 3$ of the UE were distributed in the significantly lower TSR range, where the UE were located in the lower to the lower part of the normal Uua range. UE in the normal TSR range were located in the lower to the lower part of the normal Uua range. The ratio of Uua/TSR in the UE was nearly equal to that in the NC. These findings suggest that TSR correlates highly with Uua, and low TSR is one of the main causes of urate underexcretion in UE. The majority of the OP was located in the higher part of the normal TSR and the higher Uua range. Generally, the level of reduction in the TSR in the UE compared to the NC was higher than that in UTS compared to the NC, and suggested that TSR is a better parameter for estimating underexcretion in HU. Decreased TSR was compensated for by increased Sua at the stage of urate secretion in HU.

$\mathrm{UR}_{2}$-versus-Uua Graph (fig. 6)

The distribution of the UE was spread in the horizontal $\left(\mathrm{UR}_{2}\right)$ direction from the lower to the higher $U_{2}$ range. Approximately $2 / 3$ of the UE were located in the normal $U_{2}$ range, 
Nakamura et al.: Variation of Urate Transport in the Nephrons in Subtypes of Hyperuricemia

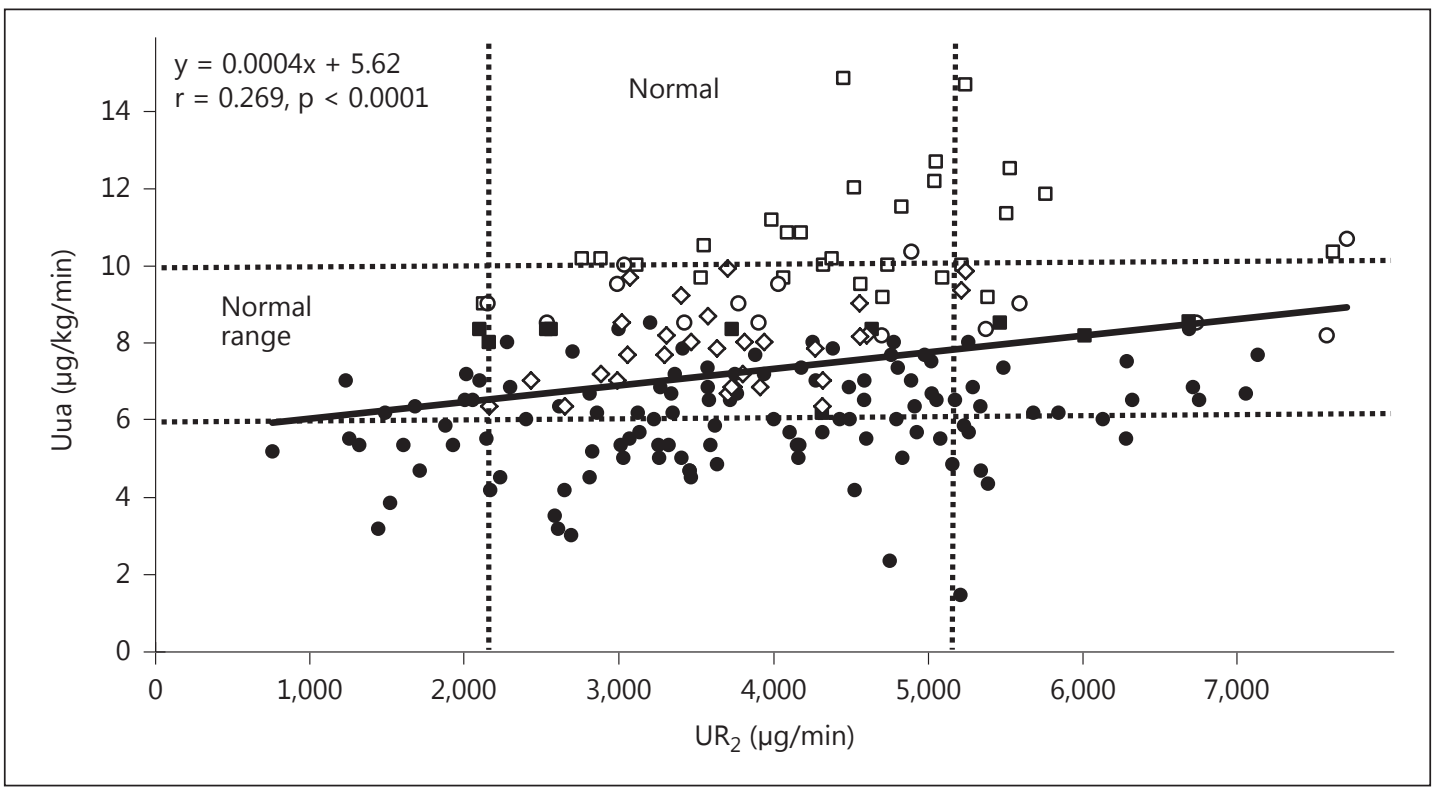

Fig. 6. Distribution of each type of $\mathrm{HU}$ on a plot evaluating Uex as a function of $\mathrm{R}_{2}$.

$=\mathrm{UE} ; \square=\mathrm{OP} ; \mathrm{O}=\mathrm{C}$; $\mathbf{\square}=\mathrm{N} ; \diamond=\mathrm{NC} ;----=$ regression equation and line.

where these UE were located in the significantly lower to the lower normal Uua range. That is, half of these UE were located in the significantly lower Uua range. The Uua/UR $\mathrm{U}_{2}$ ratio in the UE was lower than that in the $\mathrm{NC}$, suggesting that other factors except $\mathrm{UR}_{2}$ could be the main cause of urate underexcretion. A significant correlation between $U_{2}$ and Uua was noted $(r=$ $0.269, \mathrm{p}<0.00001$ ) although the coefficient value was not high.

\section{$\mathrm{R}_{2}$-versus-Uua Graph (fig. 7)}

A significant negative correlation was obtained between $R_{2}$ and Uua $(r=-0.393, p<$ $0.0001)$. The distribution of the UE was spread widely in the horizontal $\left(R_{2}\right)$ direction from the lower to the higher $R_{2}$ range, and the majority of UE were densely located in the higher $R_{2}$ region to the higher part of the normal $R_{2}$ range. Uua of these UE in the dense distribution was located in the significantly lower Uua range to the lower half of the normal range. Uua/ $R_{2}$ of the majority of the UE in the dense distribution was almost the same as that in the NC. These findings suggest that significantly higher $\mathrm{R}_{2}$ might be one of the main causes of urate underexcretion in the HU, especially in the UE. However, approximately $1 / 6$ of all UE were located in the significantly lower $\mathrm{R}_{2}$ range, and the majority of these cases were shown to have normal Uua, suggesting that the cause of urate underexcretion in UE might not always be increased reabsorption.

The OP were mainly located in the normal $\mathrm{R}_{2}$ range, with the majority of these OP shown to have significantly higher Uua than the NC. The OP with significantly lower $\mathrm{R}_{2}$ represented approximately only $1 / 4$ of all OP, and of all OP there was only 1 case (3.6\%) with significantly higher $\mathrm{R}_{2}$.

These distribution studies on various combinations of urate transport coefficients as coordinates showed that hyperuricemia could be produced by various combinations of transport abnormalities. 
Nakamura et al.: Variation of Urate Transport in the Nephrons in Subtypes of Hyperuricemia

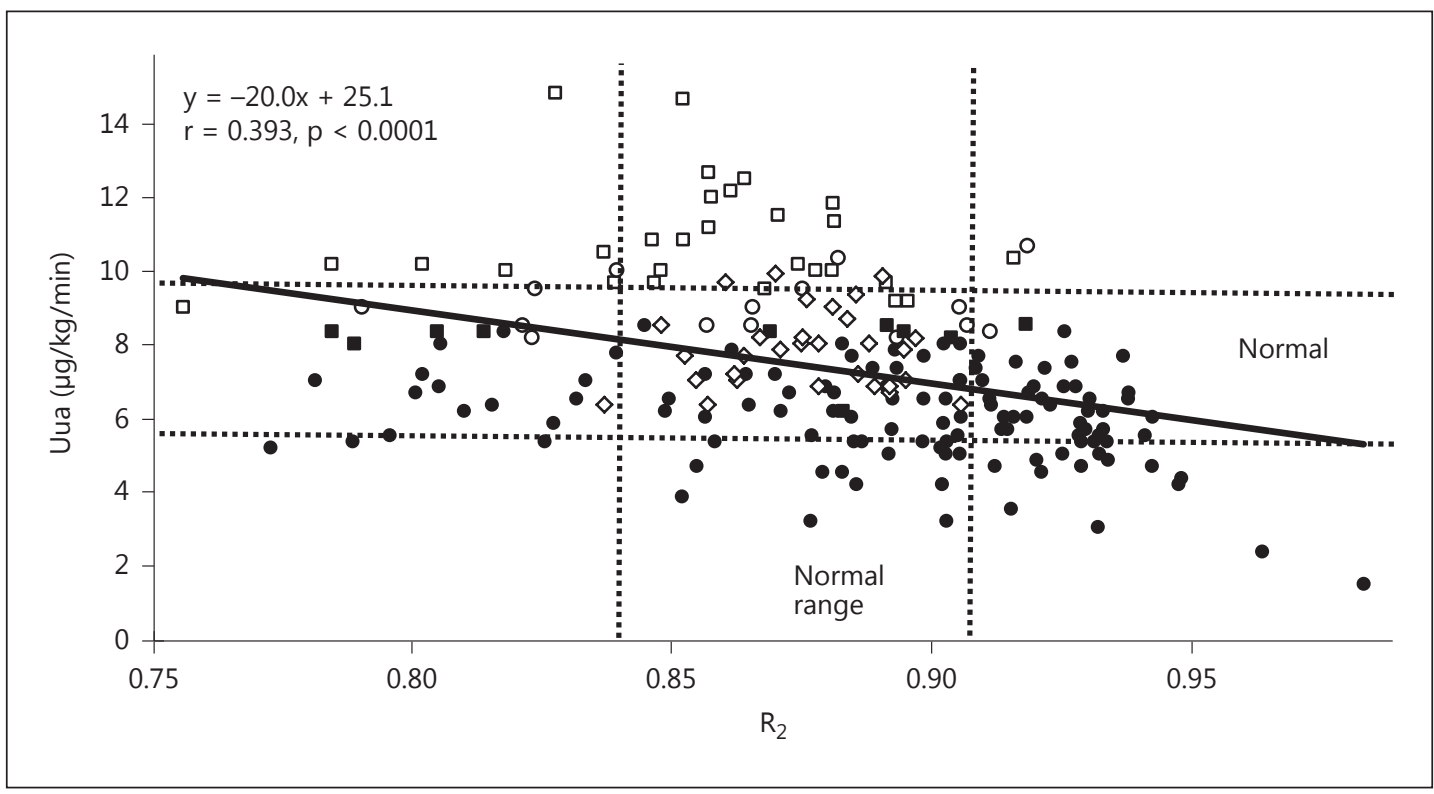

Fig. 7. Distribution of each type of $\mathrm{HU}$ on a plot evaluating Uua as a function of $\mathrm{R}_{2}$ $\mathbf{\square}=\mathrm{N} ; \diamond=\mathrm{NC} ;----=$ regression equation and line.

\section{Discussion}

In the present study, urate transport and intratubular urate contents in the tubule of nephrons in the subtypes of $172 \mathrm{HU}$ were estimated by employing a previously designed equation, $\mathrm{Cua}=\left[\mathrm{C} \operatorname{cr}\left(1-\mathrm{R}_{1}\right)+\mathrm{TSR}\right]\left(1-\mathrm{R}_{2}\right)$, using the BBR-loading urate clearance test [7]. Differences in urate transport between UE and OP were analyzed, and the relationships between urate transport and Uua were compared in the subtypes of the HU. Since the causes of hyperuricemia differ markedly between UE and OP, characteristic features of urate transport in UE and in OP might also differ markedly, reflecting differences in urate excretion and the pathophysiology of intratubular urate contents. Analysis of the differences might prove valuable in understanding the mechanisms underlying urate underexcretion in hyperuricemia.

In the subtypes of the $\mathrm{HU}$, the UE were shown to have the lowest TSR and the highest $\mathrm{R}_{2}$ in intratubular urate flow. Conversely, the OP had the highest TSR and the lowest $\mathrm{R}_{2}$. TSR and Uua showed a significant correlation $(r=0.367, p<0.0001)$, and $R_{2}$ and Uua showed a significant negative correlation $(r=-0.393, p<0.0001)$. In addition, the majority of UE were located in the significantly lower TSR range area in the TSR-versus-Uua plot and were also located in the significantly higher $\mathrm{R}_{2}$ range to the higher part of the normal range in the $\mathrm{R}_{2}$-versus-Uua plot. The Uua/TSR and Uua/ $R_{2}$ ratios of these UE in the main distribution of the graph were almost equal to the ratio of the NC. These findings suggest that TSR and $\mathrm{R}_{2}$ are closely related to Uua and that both decreased TSR and increased $\mathrm{R}_{2}$ are considered as the main causes of urate underexcretion in UE, although the effects of urate underexcretion were reduced to some extent at the following stage of UTS and $\mathrm{UR}_{2}$, respectively.

As shown in table 2, TSR and UTS were highly correlated ( $\mathrm{r}=0.773, \mathrm{p}<0.0001)$. The HU distribution pattern in the TSR-versus-Uua graph (fig. 5) and in the UTS-versus-Uua graph (fig. 4) appeared to be similar except for the distance from the NC distribution. In comparison with HU distribution in the TSR-versus-Uua plot and UTS-versus-Uua plot, the former could be shifted in a higher TSR direction to roughly replicate the latter. This finding could be under- 
stood from the following relationship of UTS and TSR: UTS $=$ TSR $\times$ Sua [7]. The distance between the distribution of $\mathrm{HU}$ and $\mathrm{NC}$ was reduced in the UTS-versus-Uua plot compared to the TSR-versus-Uua plot by multiplying higher Sua by TSR in the former. These findings suggest that decreased Uua, produced in association with the decrease of TSR in the UE compared to the NC, is improved by the product of TSR and higher Sua in the UE compared to the NC. Hyperuricemia is thus considered to represent a reasonable reaction for reducing the decreased urate excretion that might have been induced by decreased TSR. Decreased TSR can thus be considered as the primary cause of urate underexcretion in UE and offers a better parameter for estimating urate secretion abnormality in tubules than UTS.

$\mathrm{R}_{2}$ and $\mathrm{UR}_{2}$ are also highly correlated $(\mathrm{r}=0.655, \mathrm{p}<0.0001)$. Like the relationship between the distribution of the HU in the TSR-versus-Uua and UTS-versus-Uua plots, the distribution of the $\mathrm{HU}$ in the $\mathrm{R}_{2}$-versus-Uua plot (fig. 7) could be shifted in a higher $\mathrm{R}_{2}$ direction to roughly replicate that in the $U_{2}$-versus-Uua plot (fig. 6). Judging from the higher, dense distribution of the UE in the significantly higher $\mathrm{R}_{2}$ range in the $\mathrm{R}_{2}$-versus-Uua plot, $\mathrm{R}_{2}$ was considered to also be a main cause of urate underexcretion, and $R_{2}$ could offer a better parameter of urate underexcretion than $\mathrm{UR}_{2}$. Decreased TSR and increased $\mathrm{R}_{2}$ were thus considered as the main mechanisms of urate underexcretion in UE.

URAT1 has been reported as the most potent urate transporter for urate reabsorption and was highly inhibited by BBR [13]. URAT1 is also closely related to Sua in humans [14]. However, in other experiments using cultured Xenopus laevis oocytes injected with URAT1 mRNA, inhibition of urate uptake by BBR at a saturated concentration was $54.9 \%$, while the inhibition of URAT1 by BBR at the same saturated concentration level was 95.1\% [15]. These findings suggest that almost complete inhibition of URAT1 with BBR could not cause complete inhibition of all urate transport in reabsorption. For the remaining urate transport, numbers of urate transporters such as OAT1 and its families [16-25], including nicotinate transporter h0AT10 (SLC22A13) [26], fructose transporter SLC2A9 [15] and URATv1 [27], might continue to function. Another transporter, ABCG2, mediates urate secretion in proximal tubule cells, and genetic variants with single nucleotide polymorphisms were associated with high Sua [28-30]. These findings suggest that urate transporters play important roles in urate transport in the nephron and urate excretion. However, in the present study, the urate transport was assumed to be parallel with intratubular urate contents as a tentative example, and both increased $R_{2}$ and decreased TSR were suggested to be functioning independently as causes of urate underexcretion. Estimation of the grade of low TSR and high $\mathrm{R}_{2}$ in individual cases with a simple method such as the BBR-loading urate clearance test might be valuable for investigating the pathophysiology of urate transport in nephrons and controlling hyperuricemia in clinical practice.

The distribution of the HU in the $\mathrm{UR}_{2}$-versus-Uua plot (fig. 6) showed a positive correlation between $\mathrm{UR}_{2}$ and Uua. This finding seems to contradict the common concept that the correlation coefficient between $\mathrm{UR}_{2}$ and Uua would be negative. However, intratubular urate contents of the UE in the significantly higher UTS region were certainly higher than intratubular urate contents in other regions and Uua of the UE in this region was not sufficiently reduced compared to Uua in other regions of the UE after reabsorption by $U_{2}$ (fig. 4), although $\mathrm{UR}_{2}$ was relatively high because $\mathrm{UR}_{2}$ and UTS showed an extremely high correlation $(\mathrm{r}=$ $0.993, p<0.0001$ ). Therefore, increased UTS was considered to exceed the increase in $U_{2}$ and subsequently resulted in higher Uua than commonly expected.

Another 20 UE (approximately 1/6 of the 117 UE) were located in the significantly lower $\mathrm{UR}_{2}$ range in the $\mathrm{UR}_{2}$-versus-Uua plot (fig. 6) and half of these 20 cases were shown to have significantly lower Uua than seen in the NC. The latter cases were shown to have significantly lower Uua despite a low rate of urate reabsorption, suggesting that intratubular urate contents in these UE were sufficiently lower compared to the UE in other regions and the NC at the $\mathrm{UR}_{2}$ 
Nakamura et al.: Variation of Urate Transport in the Nephrons in Subtypes of Hyperuricemia

stage of intratubular urate flow. UTS and TSR in the UE thus show a wide variation and represent important factors for determining Uua in the $\mathrm{HU}$ in combination with $\mathrm{R}_{2}$.

Very high correlation coefficients between Uua and Uex $(r=0.939, p<0.0001)$ suggested that the calculation of urate transport coefficients using the equation $\mathrm{Cua}=\left[\mathrm{Ccr}\left(1-\mathrm{R}_{1}\right)+\mathrm{TSR}\right]$ $\left(1-R_{2}\right)$ is suitable. Using this equation, TSR and $R_{2}$ in the NC was calculated as $78.0 \mathrm{ml} / \mathrm{min}$ and 0.877 by substitution of $\mathrm{Cua}=9.76 \mathrm{ml} / \mathrm{min}, \mathrm{Ccr}=133 \mathrm{ml} / \mathrm{min}$ and $\mathrm{R}_{1}=0.99$, respectively [7]. Urate transporters (UGF, UTS, $\mathrm{UR}_{2}$ ) and Uex were then calculated as 7,062, 4,142, 3,694 and $519 \mu \mathrm{g} / \mathrm{min}$, respectively. Similarly, values in the UE were calculated as 8,660, 4,217, 3,890 and $414 \mu \mathrm{g} / \mathrm{min}$, respectively. These values are comparable to those in table 1 . For example, in a tentatively assumed condition where in the urate intratubular flow in the $\mathrm{NC}$, only TSR was assumed to be decreased from $78.0 \mathrm{ml} / \mathrm{min}$ in the NC to $48.7 \mathrm{ml} / \mathrm{min}$ in the UE, Cua would be calculated as $6.15 \mathrm{ml} / \mathrm{min}$ and Uex would be $321 \mu \mathrm{g} / \mathrm{min}$. Similarly, if $\mathrm{R}_{2}$ was assumed to be increased from 0.877 in the NC to 0.904 in the UE, Cua would be calculated as $7.62 \mathrm{ml} / \mathrm{min}$ and Uex would be $404 \mu \mathrm{g} / \mathrm{min}$. The marked decreases in Uex by the one transporter change suggest that both decreased TSR and increased $\mathrm{R}_{2}$ are fundamental factors for urate underexcretion in UE.

\section{Conclusion}

Both TSR and $\mathrm{R}_{2}$ represent fundamental factors for determining Uua in HU. Significantly lower TSR and significantly higher $\mathrm{R}_{2}$ were considered as the main causes of urate underexcretion in UE. Neither was apparent in OP. Understanding the difference between UE and OP in terms of the pathophysiology of urate transport in urate underexcretion might be useful for controlling hyperuricemia in clinical practice.

\section{Acknowledgements}

This work was supported in part by a Grant-in-Aid from the Gout Research Foundation of Japan. We wish to thank Prof. Akira Nakamura from the Institute of Higher Education Research and Practice, Osaka University, for useful discussions regarding mathematical calculations, Mr. Naoki Okabayashi and Mr. Takeshi Tazima from Hayashi General Hospital for their assistance with computer graphic figure formation, and Ms. Noriko Tamezawa and Ms. Naomi Hamano from Hayashi General Hospital for their technical assistance in BBRloading urate clearance tests.

\section{References}

1 Seegmiller JE, Grayzel AI, Laster L, Liddle L: Uric acid production in gout. J Clin Invest 1961;40:1304-1314.

2 Benedict JD, Forsham PH, Stetten D Jr: The metabolism of uric acid in the normal and gouty human studied with the aid of isotopic uric acid. J Biol Chem 1949;181:183-193.

3 Pallella TD, Fox IH: Hyperuricemia and gout; in Sciver CR, Beaudet AL, Sly WWS, Valle D (eds): The Metabolic Basis of Inherited Disease. New York, McGraw-Hill, 1989, pp 965-1006.

4 Wyngaarden JE: Overproduction of uric acid as the cause of hyperuricemia in primary gout. J Clin Invest 1957; 36:1508-1515.

5 Nakamura T, Uchida M, Uchino H, Higuchi T: Studies on hyperuricemia of gout by means of the uric acid clearance. Uric Acid Res 1977;1:45-61.

6 Imamura S, Nakamura T: Characteristic features in pathophysiology of hyperuricemia with overproduction in gout. Purine Pyrimidine Metab 1994;18:132-143. 
Nakamura et al.: Variation of Urate Transport in the Nephrons in Subtypes of Hyperuricemia

7 Nakamura T, Nishi R, Yamashita T, Tanaka T, Takagi K, Yamashita T, Yamauchi T, Ueda T: Quantitative estimation of urate transport in nephrons in relation to urinary excretion employing benzbromarone loading urate clearance tests in cases of hyperuricemia. Nephron Extra 2011;1:55-68.

8 Nakamura T, Tanaka T, Takagi K, Nakayama T, Inai K, Tsutani H, Ueda T: Urate transport in nephrons of gouty patients: quantitative analysis of urate transport in nephrons. Clin Exp Nephrol 1999;3:169-174.

-9 Kamatani N, Fujimori S, Hada T, Hosoya T, Kohri K, Nakamura T, Ueda T, Yamamoto T, Yamanaka H, Matsuzawa Y: Multicenter, open-label study of long-term administration of febuxostat (TMX-67) in Japanese patients with hyperuricemia including gout. J Clin Rheumatol 2011;17:S50-S56.

10 Becker MA, MacDonald PA, Hunt B, Gunawardhana L: Treating hyperuricemia of gout: safety and efficacy of febuxostat and allopurinol in older versus younger subjects. Nucleosides Nucleotides Nucleic Acids 2011;30: 1011-1017.

11 Nakamura T: Studies on uric acid metabolism in hyperuricemia. Jpn J Clin Med 1973;31:1161-1172.

12 Nakamura T, Uchida M, Uchino H, Higuchi T: Studies on hyperuricemia of gouty patients employing uric acid clearance tests. Uric Acid Res 1978;2:125-130.

-13 Enomoto A, Kimura H, Chairoungdua A, Shigeta A, Jutabha P, Cha SH, Hosoyamada M, Takeda M, Sekine T, Igarashi T, Matsuo H, Kikuchi Y, Oda T, Ichida K, Hosoya T, Shimokata K, Niwa T, Kanai Y, Endou H: Molecular identification of a renal urate-anion exchanger that regulates blood urate levels. Nature 2002;417:447-452.

14 Ichida K, Hosoyamada M, Hisatome I, Enomoto A, Hikita M, Endou H, Hosoya T: Clinical and molecular analysis of patients with renal hypouricemia in Japan - influence of URAT1 gene on urinary urate excretion. J Am Soc Nephrol 2004;15:164-173.

15 Vitart V, Rudan I, Hayward C, Gray NK, Floyd J, Palmer CN, Knott SA, Kolcic I, Polasek O, Graessler J, Wilson JF, Marinaki A, Riches PL, Shu X, Janicijevic B, Smolej-Narancic N, Gorgoni B, Morgan J, Campbell S, Biloglav Z, Barac-Lauc L, Pericic M, Klaric IM, Zgaga L, Skaric-Juric T, Wild SH, Richardson WA, Hohenstein P, Kimber CH, Tenesa A, Donnelly LA, Fairbanks LD, Aringer M, McKeigue PM, Ralston SH, Morris AD, Rudan P, Hastie ND, Campbell H, Wrigh AF: SLC2A9 is a newly identified urate transporter influencing serum urate concentration, urate excretion and gout. Nat Genet 2008;40:437-442.

-16 Sweet DH, Wolff NA, Pritchard JB: Expression cloning and characterization of ROAT1. The basolateral organic anion transporter in rat kidney. J Biol Chem 1997;272:30088-30095.

17 Sekine T, Watanabe N, Hosoyamada M, Kanai Y, Endou H: Expression cloning and characterization of a novel multispecific organic anion transporter. J Biol Chem 1997;272:18526-18529.

18 Wolff NA, Werner A, Burkhardt S, Burckhardt G: Expression cloning and characterization of a renal organic anion transporter from winter flounder. FEBS Lett 1997;417:287-291.

19 Sekine T, Cha SH, Tsuda M, Apiwattanakul N, Nakajima N, Kanai Y, Endou H: Identification of multispecific organic anion transporter 2 expressed predominantly in the liver. FEBS Lett 1998;429:179-182.

-20 Kusuhara H, Sekine T, Utsunomiya-Tate N, Tsuda M, Kojima R, Cha SH, Sugiyama Y, Kanai Y, Endou M: Molecular cloning and characterization of a new multispecific organic anion transporter from rat brain. J Biol Chem 1999;274:13675-13680.

-21 Cha SH, Sekine T, Kusuhara H, Yu E, Kim JY, Kim DK, Sugiyama Y, Kanai Y, Endou H: Molecular cloning and characterization of multispecific organic anion transporter 4 expressed in the placenta. J Biol Chem 2000;275: 4507-4512.

22 Youngblood GL, Sweet DH: Identification and functional assessment of the novel murine organic anion transporter OAT5 (SLC22A19) expressed in kidney. Am J Physiol 2004;287:F236-F244.

-23 Schnabolk GW, Youngblood GL, Sweet DH: Transport of estrone sulfate by the novel organic anion transporter OAT6 (SLC22A20). Am J Physiol Renal Physiol 2006;291:F314-F321.

-24 Shin HJ, Anzai N, Enomoto A, He X, Kim DK, Endou H, Kanai Y: Novel liver-specific organic anion transporter OAT7 that operates the exchange of sulfate conjugates for short chain fatty acid butyrate. Hepatology 2007; 45:1046-1055.

-25 Jutabha P, Kanai Y, Hosoyamada M, Chairoungdua A, Kim DK, Iribe Y, Babu E, Kim JY, Anzai N, Chatsudthipong $\mathrm{Y}$, Endou H: Identification of a novel voltage-driven organic anion transporter present at apical membrane of renal proximal tubule. J Biol Chem 2003;278:27930-27938.

-26 Bahn A, Hagos Y, Reuter S, Balen D, Brzica H, Krick W, Burchardt BC, Sabolic I, Burckhardt G: Identification of a new urate and high affinity nicotinate transporter, hOAT10 (SLC22A13). J Biol Chem 2008;283:1633216341.

-27 Anzai N, Ichida K, Jutabha P, Kimura T, Babu E, Jin CJ, Srivastava S, Kitamura K, Hisatome I, Endou H, Sakurai $\mathrm{H}$ : Plasma urate level is directly regulated by a voltage-driven urate efflux transporter URATv1 (SLC22A9) in humans. J Biol Chem 2008;283:26834-26838.

28 Woodward OM, Koettgen A, Coresh J, Boerwinkle E, Guggino WB, Koettgen M: Identification of a urate transporter, ABCG2, with a common functional polymorphism causing gout. Proc Natl Acad Sci USA 2009;106: 10338-10342.

29 Anzai N, Endou H: Urate transporters: an evolving field. Semin Nephrol 2011;31:400-409.

30 Choi HK, Zhu Y, Mount DB: Genetics of gout. Curr Opin Rheumatol 2010;22:144-151. 